Received 7th May 2019, Accepted 00th January 20xx DOI: $10.1039 / x 0 x \times 00000 x$

\title{
Network topology and cavity confinement-controlled diastereoselectivity in cyclopropanation reactions catalyzed by porphyrin-based MOFs
}

\author{
Konstantin Epp, ${ }^{a}$ Bart Bueken, ${ }^{b}$ Benjamin J. Hofmann, ${ }^{c}$ Mirza Cokoja, ${ }^{a}$ Dirk De Vos ${ }^{b}$ and Roland A. \\ Fischer ${ }^{* a}$
}

\begin{abstract}
In this work, we show that the stereoselectivity of a reaction can be controlled by directing groups of substrates, by network topology and by local cavity confinement of metal-organic framework (MOF) catalysts. We applied the porphyrin-based PCN-224(Rh), which contains no stereocenters in the cyclopropanation reaction using ethyl diazoacetate (EDA) as carbene source. When styrene and other non-coordinating olefins are used as substrates, high activity, but no diastereoselectivity is observed. Interestingly, conversion of 4-amino- and 4-hydroxystyrene substrates occurs with high diastereomeric ratios ( $d r$ ) of up to 23:1 (trans:cis). We attribute this to local pore confinement effects as a result of substrate coordination to neighboring Rh-centers, which position the olefin with respect to the active site, causing a break of local symmetry of the coordinated substrate. The effect of local pore confinement was improved by using PCN-222(Rh) as catalyst, which is a structural analog of $\mathrm{PCN}-224(\mathrm{Rh})$ with characteristic Kagomé topology featuring shorter Rh-Rh distances. A remarkable $d r$ of 42:1 (trans:cis) was observed for 4-aminostyrene. In this case, the length of the substrate corresponds to the average distance between two neighboring Rh centers within the pores of PCN-222(Rh), which drastically boosts the diastereoselectivity. This work showcases how diastereomeric control can be achieved by favorable substrate-catalyst interactions and thoughtful adjustment of confined reaction space using porphyrin-based MOFs, in which stereocenters are inherently absent.
\end{abstract}

\section{Introduction}

Stereoselective catalysis is a key technology due to its enormous economic relevance towards the production of pharmaceuticals, agrochemicals, fungicides, pheromones, flavors and fragrances. ${ }^{1}$ In stereoselective organometallic catalysis, one of the most exploited design principles is the 'catalyst control', i.e. the coordination of catalytically active metal centers by chiral ligands to drive stereoselectivity, ${ }^{2}$ such as diamines or diphosphines, as for instance in the well-known BINAP, ${ }^{3}$ or chiral multidentate carboxylates, as is the case with the Sharpless epoxidation catalyst. ${ }^{4}$ The design of heterogeneous stereoselective catalysts is, however, more intricate. In heterogeneous catalysts, the stereoselectivity usually originates from a molecular catalyst immobilized on a

\footnotetext{
a. Konstantin Epp, Mirza Cokoja and Roland A. Fischer, Chair of Inorganic and Metal-Organic Chemistry, Catalysis Research Center and Department of Chemistry, Technical University of Munich, Ernst-Otto-Fischer-Straße 1, D-85748 Garching bei München, Germany.

b. Bart Bueken and Dirk De Vos, Centre for Surface Chemistry and Catalysis, Department of Microbial and Molecular Systems ( $\left.M^{2} S\right)$, KU Leuven, Celestijnenlaan 200F p.o. box 2461, 3001 Leuven, Belgium.

Benjamin J. Hofmann, Molecular Catalysis, Catalysis Research Center and Department of Chemistry, Technical University of Munich, Ernst-Otto-FischerStraße 1, D-85748 Garching bei München, Germany.

+ Footnotes relating to the title and/or authors should appear here.

Electronic Supplementary Information (ESI) available: [details of any supplementary information available should be included here]. See DOI: 10.1039/x0xx00000x
}

solid support. The high tuneability of metal-organic frameworks (MOFs) as heterogeneous catalysts allows much versatility in tailoring and manipulating their structural and chemical properties. In the context of stereoselective catalysis, MOF design approaches involve either the heterogenization of stereoselective molecular catalyst to the MOF linker, ${ }^{5}$ or using linkers $^{6}$ that themselves act as stereoselective ligands to catalytically active metals. ${ }^{7,8}$

While the classic 'catalyst-controlled' concept for both homogeneous and heterogenized molecular catalysts requires well-defined stereocenters, very few reports describe the stereoselectivity of solid catalyst@host materials, in which neither the catalyst nor the host contain such well-defined stereocenters. In this case, the induction of stereoselectivity is derived from pore confinement effects of the host. While this type of stereocontrol is known for soluble, defined macromolecular cage compounds and others, 9,10 only a handful examples using solid/polymeric porous materials such as zeolites, $^{11,12}$ mesoporous silica ${ }^{13,14}$ or MOFs $^{15-18}$ are known. 'Confinement' or 'confinement effects' are rather broad terms and not explicitly specified in literature. Hence, it is not exactly clear how the confinement of a host defines the stereochemical outcome of a reaction, since the local substrate arrangement through its interaction within the host are a multi-component problem. ${ }^{19}$ In many homogeneous catalysis reactions, the stereo- or regioselectivity can be influenced by interactions of 'directing' functional groups of the substrate with the catalyst, 
e.g. via hydrogen-, covalent- or coordinative bonds, or Coulomb or Lewis acid-base interactions, which induce a preferential conformation or orientation of the substrate at the catalyst. ${ }^{20}$ Therefore, it is reasonable to assume that directing effects of functional groups attached to substrates may also play a crucial role in MOF-based catalysis by coordination to suitable sites located within the pores. A thorough understanding of the origin of stereocontrol in these cases is of great interest, as the insights gained could enable further control over confinement effects in MOF-based catalysts.

As we have been working with metalloporphyrin (MP) MOFs as catalysts for the cycloaddition of carbon dioxide to propylene oxide to form propylene carbonate, ${ }^{21}$ we extended their application as heterogeneous catalysts to (dia)stereoselective reactions. Molecular MPs have been widely studied in literature, because of their ability to act as ligands for various metals (M = Fe(II,III), ${ }^{22,23} \mathrm{Co}(\mathrm{II}),{ }^{24} \mathrm{Rh}(\mathrm{III}),{ }^{25-27} \mathrm{Ru}(\mathrm{II}),{ }^{28} \operatorname{Ir}(\mathrm{III}),{ }^{29}$ Os(III) ${ }^{30}$ and others). Thus, it is not surprising that their chemistry and catalytic properties are rich. ${ }^{31,32}$ To be used in stereoselective catalysis, MPs are often functionalized with bulky ${ }^{26,33}$ and/or chiral groups ${ }^{34-36}$ at the meso-positions of the porphyrin backbone in order to induce stereochemical or even chiral information and most importantly, to engineer a pocketlike environment around the catalytically active MP center. On the one hand catalytic activities may increase to TOFs > $100,000 \mathrm{~h}^{-1}$ as demonstrated by Gallo et al. using 'totem'shaped Fe(III)-porphyrins in the cyclopropanation of styrene, ${ }^{33}$ while on the other hand synthetic yields of these sophisticated MPs are extremely low (often $<1 \%$ ) and purification is necessary to isolate the compounds. Moreover, deactivation via dimerization may hinder their application in catalysis.

Motivated by the remarkable catalytic properties of molecular MPs and the challenges in their synthesis, stability and recyclability, we were interested to investigate how their heterogeneous counterparts, namely, MP-MOFs, would perform as catalysts. Heterogenization is achieved when MPs are built into the structure of the framework as organic linkers, as in the Zr-MOFs PCN-222, 37 PCN-22438 and MOF-525.39 Consequently, MP-MOFs can be seen as rigid, self-supported heterogeneous catalysts with well-defined, spatially distributed MP-sites, which are catalytically active and not susceptible to dimerization. Thus, here we investigate for the first time how different MP-MOFs phases, PCN-224(Rh) and PCN-222(Rh), constructed from the same building blocks, i.e. $\mathrm{Zr}_{6}$ inorganic nodes and MP linkers, but with different topologies influence the stereoselectivity of a catalytic reaction. The utilization of unaltered building units, especially the MP linkers as the catalytically active sites, allows direct comparison of the catalytic results. Hence, the difference in stereoselectivity can be attributed to the different confinement effects of altered pore sizes and geometries.

Since MPs are used in the cyclopropanation of olefins, we choose this as a reaction. Cyclopropane motifs are not only found in natural products; they are also important synthetic targets in pharmaceuticals like antibiotics, in perfume compounds and in biomimetic insect repellents. ${ }^{40,41}$ The specific reactivity of the strained three-membered ring system and its ability to induce conformational constraints on otherwise flexible acyclic chains are key features of this motif. ${ }^{41-43}$ The transition-metal catalyzed cyclopropanation of double bonds using diazo compounds as a carbene source is well studied and has been recognized as a useful design route for substituted cyclopropanes. Since there is a vast number of reports using $\mathrm{Rh}$ (II,III) catalysts in the CP reaction, ${ }^{25-27,44,45}$ we decided to start with the $\mathrm{Rh}$ (III)-metalated porphyrin, namely $[5,10,15,20$ tetrakis(4-methoxycarbonylphenyl)porphyrinato]-Rh(III) chloride $\left(\mathrm{Rh}\left(\mathrm{TCPPCO}{ }_{2} \mathrm{Me}\right) \mathrm{Cl}\right)$ as a homogeneous reference system in the cyclopropanation of styrene with ethyl diazoacetate (EDA) to the corresponding cyclopropanation products (trans and cis). Consecutively, we compared these results with those obtained on stable heterogeneous MP-based PCN-224(Rh) and PCN-222(Rh) catalysts with regard to activity and $d r$.

\section{Experimental section}

\subsection{Materials and Methods}

All chemicals were purchased from commercial sources and used without any purification. Benzoic acid, benzonitrile, cyclooctene, 4-nitrostyrene, 4-tert-butylstyrene, ethyl diazoacetate (EDA) and 4-hydroxystyrene were purchased from Sigma Aldrich, $\mathrm{RhCl}_{3}$ from Precious Metals Online, methyl 4formylbenzoate from Merck, pyrrole, styrene, 1-octene, 4chlorostyrene, 3,4-dihydro-2H-pyran and $\mathrm{N}, \mathrm{N}$ diethylformamide (DEF) from TCl, propionic acid, and $N, N$ dimethylformamide (DMF), $\mathrm{N}, \mathrm{N}$-dimethyl-4-aminostyrene from abcr, $\mathrm{ZrCl}_{4}$, from Alfa Aesar.

The Rh linker was synthesized with slight changes to the published procedures. ${ }^{46}$ The Rh-porphyrin ester $\mathrm{Rh}\left(\mathrm{TCPPCO}_{2} \mathrm{Me}\right) \mathrm{Cl}$ was prepared by mixing $\mathrm{TCPPCO}_{2} \mathrm{Me}(168.2$ $\mathrm{mg}, 0.2 \mathrm{mmol})$ and $\mathrm{RhCl}_{3}(104.2 \mathrm{mg}, 0.8 \mathrm{mmol})$ in benzonitrile $(5 \mathrm{~mL})$ under stirring at $220^{\circ} \mathrm{C}$ for $2 \mathrm{~h}$. Benzonitrile was removed under reduced pressure and the residue was dried at $80{ }^{\circ} \mathrm{C}$ overnight. The resulting $\mathrm{Rh}\left(\mathrm{TCPPCO}_{2} \mathrm{Me}\right) \mathrm{Cl}$ was hydrolyzed $(120$ $\mathrm{mg}$ ) by refluxing a mixture of THF $(6 \mathrm{~mL})$ and $\mathrm{MeOH}(6 \mathrm{~mL})$, to which a solution of $\mathrm{KOH}(100 \mathrm{mg})$ in $\mathrm{H}_{2} \mathrm{O}(6 \mathrm{~mL})$ was added within $5 \mathrm{~h}$. After the reaction mixture was cooled down to room temperature, the organic solvents were removed by rotary evaporation. Additional water was added to the residual product mixture and acidified with $1 \mathrm{M} \mathrm{HCl}$ until no further precipitation of $\mathrm{Rh}\left(\mathrm{TCPPCO}_{2} \mathrm{H}\right) \mathrm{Cl}$ was observed. The red product was washed with water $(3 \times 50 \mathrm{~mL})$ and dried in vacuum overnight.

\section{Powder X-Ray Diffraction (PXRD)}

Powder X-ray diffraction measurements were performed using Bragg-Brentano geometry in a PANalytical Empyrean diffractometer equipped with a PANalytical PIXcel 1D detector. $X$-ray Cu K $\alpha$ radiation $(\lambda 1=1.5406 \AA, \lambda 2=1.5444 \AA, 12 / 11=0.5)$ was used for the measurements. $K \beta$ radiation removed with a $\mathrm{Ni}$ filter. Voltage and intensity were $45 \mathrm{kV}$ and $40 \mathrm{~mA}$, respectively. The measurement range was from $5.0^{\circ}$ to $70.0^{\circ}$ (20) with a step size of $0.040^{\circ}(2 \theta)$ and an acquisition time of 35 seconds per step. The measurement was performed at $298 \mathrm{~K}$ and the sample was rotated on a reflection-transmission 
spinner during the measurement (0.5 rps). High-throughput powder X-ray diffraction patterns were recorded on a STOE COMBI P diffractometer (monochromated $\mathrm{Cu} \mathrm{K}_{\alpha 1}$ radiation, $\lambda=$ $1.54060 \AA$ ̊) equipped with an IP-PSD detector in transmission geometry.

\section{$\mathrm{N}_{2}$-Physisorption (Brunauer-Emmett-Teller, BET)}

Nitrogen physisorption measurements were performed on a Micromeritics 3Flex surface analyzer and carried out at $77 \mathrm{~K}$. Prior to the measurements, the samples ( 100 mg) were activated for $12 \mathrm{~h}$ at $393 \mathrm{~K}$ in vacuum. The specific surface area of the materials was calculated using the multipoint BET method (Brunauer-Emmett-Teller) applied to the isotherm adsorption branch, while taking into account the Rouquerol consistency criteria. ${ }^{47}$

\section{NMR spectra}

${ }^{1} \mathrm{H}$ and ${ }^{13} \mathrm{C}$ NMR spectra were recorded on a Bruker AMX-300 spectrometer at $300 \mathrm{MHz}$ and $75 \mathrm{MHz}$, respectively (16 and 1024 scans, respectively).

\section{UV-VIS}

UV-VIS spectroscopy was performed on an Agilent Cary 60 UVVIS instrument.

\section{Material synthesis}

Synthesis of PCN-224. In a Pyrex vial $\mathrm{ZrCl}_{4}(30 \mathrm{mg}), \mathrm{H}_{2} \mathrm{TCPP}$ $(10 \mathrm{mg})$ and benzoic acid $(400 \mathrm{mg})$ were ultrasonically dissolved in DMF ( $2 \mathrm{~mL}$ ). The mixture was heated in an oven at $120^{\circ} \mathrm{C}$ for $24 \mathrm{~h}$. After cooling down to room temperature, purple crystals were collected by filtration, washed with DMF (3x $20 \mathrm{~mL})$ and acetone ( $3 \times 20 \mathrm{~mL}$ ), and placed in acetone solution for additional $24 \mathrm{~h}$ to ensure sufficient solvent exchange. The powder was separated by centrifugation and activated at $120^{\circ} \mathrm{C}$ for $24 \mathrm{~h}$ in dynamic vacuum. Anal. Calcd. (\%) for PCN-224: C, 42.01; H, 2.45; N, 4.08\%. Found: C, 44.51; H, 2.74; N, 3.77\%.

Synthesis of PCN-224(Rh). In a Pyrex vial $\mathrm{ZrCl}_{4}$ (30 mg), $\mathrm{Rh}\left(\mathrm{TCPPCO}_{2} \mathrm{H}\right) \mathrm{Cl}(10 \mathrm{mg})$ and benzoic acid $(400 \mathrm{mg})$ were ultrasonically dissolved in DMF ( $2 \mathrm{~mL})$. The mixture was heated in an oven at $120{ }^{\circ} \mathrm{C}$ for $24 \mathrm{~h}$. After cooling down to room temperature, orange-red crystals were collected by filtration, washed with DMF (3x $20 \mathrm{~mL})$ and acetone ( $3 \times 20 \mathrm{~mL})$, and transferred in acetone solution for $24 \mathrm{~h}$. The powder was separated by centrifugation and activated at $120^{\circ} \mathrm{C}$ for $24 \mathrm{~h}$ in dynamic vacuum. Anal. Calcd. (\%) for PCN-224(Rh): C, 39.49; $\mathrm{H}$, 2.16; N, 3.84\%; Found: C, 40.20; H, 3.01; N, 3.92\%.

Synthesis of PCN-222. In a Pyrex vial $\mathrm{ZrCl}_{4}\left(70 \mathrm{mg}\right.$ ), $\mathrm{H}_{2}$ TCPP (50 $\mathrm{mg}$ ), and benzoic acid ( $2.7 \mathrm{~g}$ ) were ultrasonically dissolved in DMF $(8 \mathrm{~mL})$. The mixture was heated in an oven at $120^{\circ} \mathrm{C}$ for 2 d. After cooling down to room temperature, violet powder was collected by filtration and activated with $1.5 \mathrm{~mL} 8 \mathrm{M} \mathrm{HCl}$ in DMF at $120^{\circ} \mathrm{C}$ for $12 \mathrm{~h}$ to remove residual modulator, followed by washing with DMF (3x $20 \mathrm{~mL})$ and acetone $(3 \times 20 \mathrm{~mL})$ and transferred into acetone solution for $24 \mathrm{~h}$. The powder was separated by centrifugation and activated at $120^{\circ} \mathrm{C}$ for $24 \mathrm{~h}$ in dynamic vacuum. Anal. Calcd. (\%) for PCN-222: C, 48.27; H, 2.70; $\mathrm{N}, 4.69 \%$. Found: $\mathrm{C}, 46.36 ; \mathrm{H}, 2.68 ; \mathrm{N}, 5.40 \%$.

Synthesis of PCN-222(Rh). In a Pyrex vial $\mathrm{ZrCl}_{4}$ (70 mg), $\mathrm{Rh}(\mathrm{TCPP}) \mathrm{Cl}(50 \mathrm{mg}$ ) and benzoic acid $(2.7 \mathrm{~g}$ ) were ultrasonically dissolved in DMF ( $8 \mathrm{~mL}$ ). The mixture was heated in an oven at $120{ }^{\circ} \mathrm{C}$ for $2 \mathrm{~d}$. After cooling down to room temperature, the resulting dark red powder was collected by filtration, washed with DMF (6x $20 \mathrm{~mL})$ and acetone $(6 \times 20 \mathrm{~mL})$ and transferred into acetone solution for $48 \mathrm{~h}$. During that time, acetone was exchanged every 12 hours. The powder was separated by centrifugation and activated at $120{ }^{\circ} \mathrm{C}$ for $24 \mathrm{~h}$ in dynamic vacuum. Anal. Calcd (\%) for PCN-222(Rh): C, 44.94; H, 2.36; N, 4.28\%; Found: $C, 46.90 ; \mathrm{H}, 2.66 ; \mathrm{N}, 4.41 \%$.

\subsection{Synthesis and Characterisation}

PCN-224(Rh) was obtained by solvothermal synthesis. A metalated Rh-porphyrin linker ${ }^{46}$ was synthesized by saponification of the $\mathrm{Rh}\left(\mathrm{TCPPCO}_{2} \mathrm{Me}\right) \mathrm{Cl}$ ester. Metalation was confirmed via UV-Vis spectroscopy (see the Electronic Supplementary Information (ESI), Figure S1). The obtained linker $\quad[5,10,15,20$-tetrakis(4carboxyphenyl)porphyrinato] $\mathrm{Rh}$ (III) chloride, $\mathrm{ZrCl}_{4}$ and benzoic acid as modulator were mixed in DMF and heated for 1 day at $120^{\circ} \mathrm{C} .{ }^{38}$. Accordingly, $\mathrm{PCN}-222(\mathrm{Rh})$ was synthesized utilizing lower $\mathrm{Zr}$ : linker ratios and extended reaction tines. ${ }^{38}$

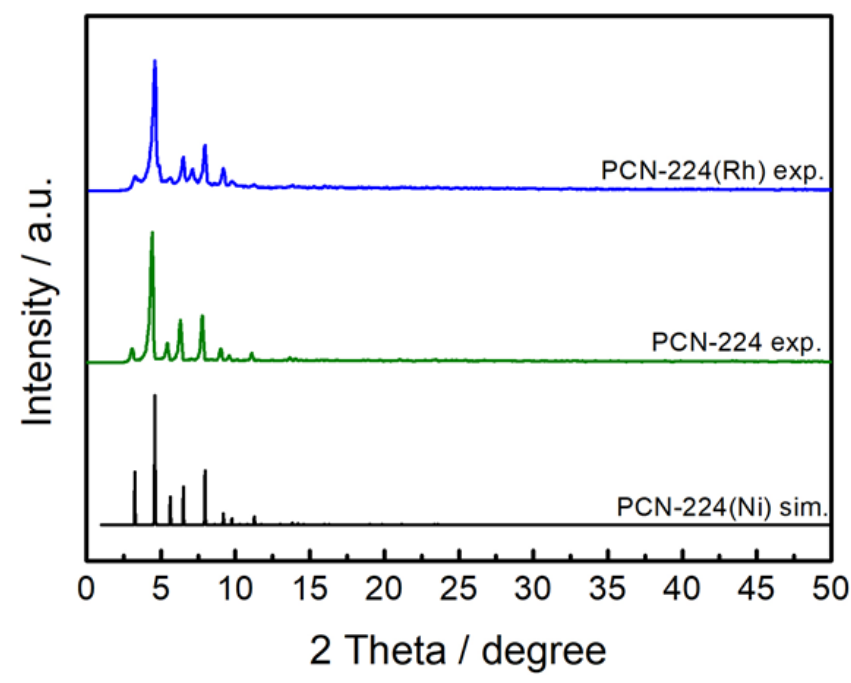

Figure 1. Experimental (exp.) powder X-ray diffraction pattern of $\mathrm{PCN}-224(\mathrm{Rh}, \mathrm{Fe}$ compared to non-metalated exp. PCN-224 and the simulated (sim.) pattern of $\mathrm{PCN}-224(\mathrm{Ni})$ calculated from a model obtained from single crystal data.

MP-MOFs were obtained as phase pure microcrystalline powders, which was confirmed by powder X-ray diffraction (PXRD) (see Figure S2 in the ESI for the PXRD of PCN-222(Rh)). Comparison to patterns of parent non-metalated MOFs and to the simulated powder pattern reveals that all MP-MOFs are isostructural to each other. A type $\mathrm{I}$ isotherm and a Brunauer-Emmett-Teller (BET) surface area (Sa) of $1400 \mathrm{~m}^{2} / \mathrm{g}$ were found for PCN-224(Rh). This value is lower than, but still in the same order of magnitude as for non-metalated PCN-224 (Sa $=2147 \mathrm{~m}^{2} / \mathrm{g}$ ). This might indicate slightly incomplete activation prior to the measurement, or possibly the presence of additional Rh-ions, which would increase the molar weight but hardly affect the pore volume (see the ESI for the pore size distributions (PSD)). 


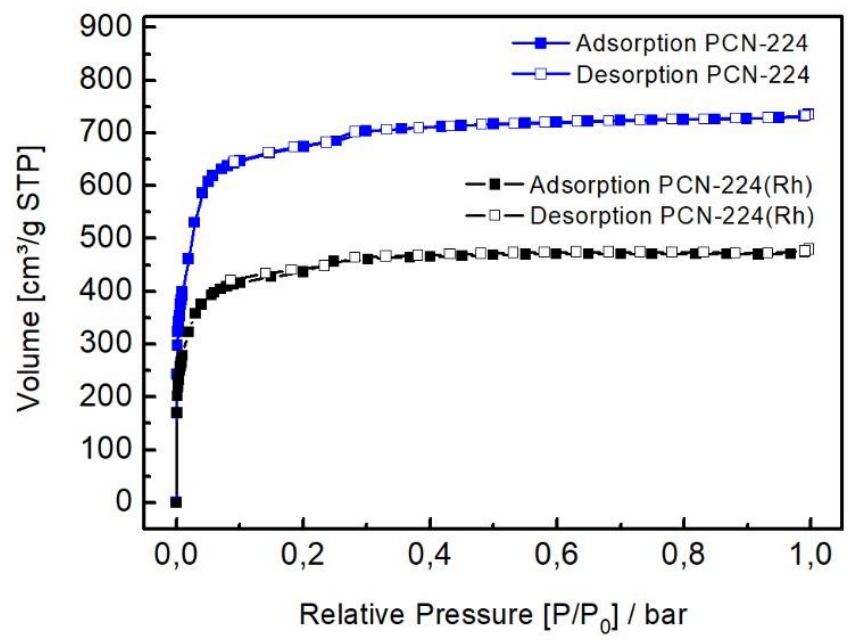

Figure 2. $\mathrm{N}_{2}$-physisorption measurements of PCN-224(Rh, no metal) measured at $77 \mathrm{~K}$.

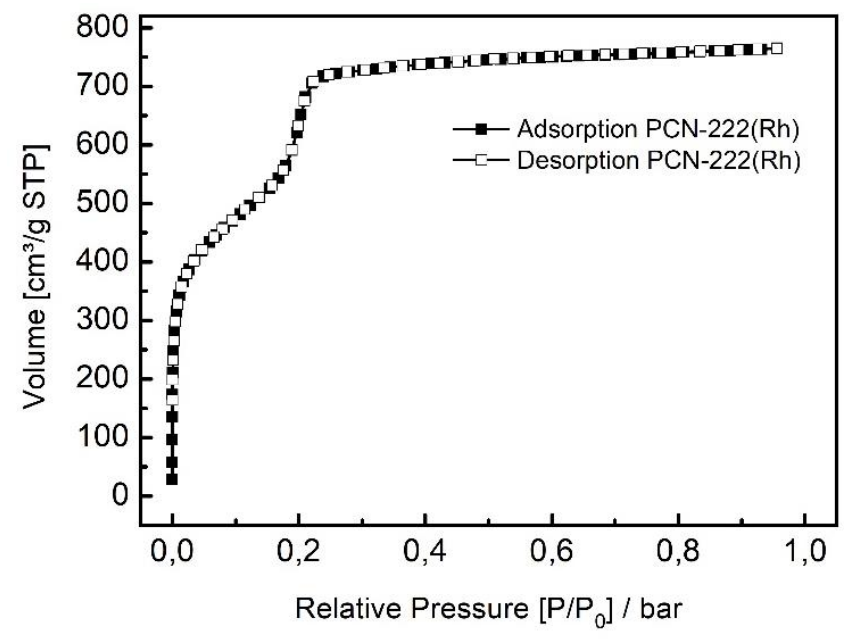

Figure 3. $\mathrm{N}_{2}$-physisorption measurements of $\mathrm{PCN}-222(\mathrm{Rh})$ measured at $77 \mathrm{~K}$.

The synthesized micro- and mesoporous PCN-222(Rh) sample exhibited a surface area of $1912 \mathrm{~m}^{2} / \mathrm{g}$ (literature Sa of nonmetalated PCN-222 = $\left.2223 \mathrm{~m}^{2} / \mathrm{g}\right),{ }^{37}$ showing an expected additional step in the isotherm compared to PCN-222 which could be attributed to the large hexagonally shaped 3D mesoporous channels (see the ESI for the pore size distribution of PCN-222(Rh)).

\subsection{Catalytic tests}

As a test reaction, we selected the cyclopropanation (CP) of olefins (see Scheme 1) using ethyl diazoacetate (EDA) as carbene source.

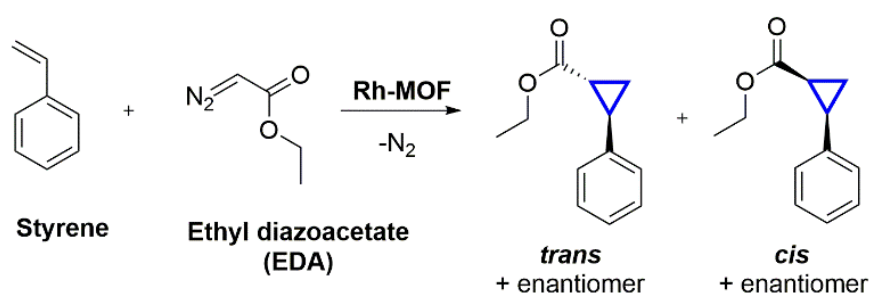

Scheme 1. Cyclopropanation of styrene using ethyl diazoacetate (EDA) to the corresponding diastereomeric cyclopropanation products (trans, cis and their corresponding enantiomers (not shown)) under Rh-MOF catalysis.

First, we tested $\mathrm{Rh}\left(\mathrm{TCPPCO}_{2} \mathrm{Me}\right) \mathrm{Cl}$ as a homogeneous reference system. In the catalytic reaction $135 \mu \mathrm{L}$ EDA ( $1.1 \mathrm{mmol})$ was diluted in $2 \mathrm{~mL} \mathrm{CH} \mathrm{CH}_{2}$ and added manually via a syringe to a solution of $75 \mu \mathrm{L}$ styrene $(0.8 \mathrm{mmol})$ in $3 \mathrm{~mL} \mathrm{CH} \mathrm{Cl}_{2}$ within 20 min. Further manual introduction of EDA did not lead to full conversion. The activity at room temperature (r.t.) under stirring in $\mathrm{CH}_{2} \mathrm{Cl}_{2}$ is moderate (yield $=20 \%$ after $22 \mathrm{~h}$; see Table 1). Expectedly, there is no diastereoselectivity (1:1), since this porphyrin ligand is nearly planar and lacks directing bulky groups attached at the meso-position of the porphyrin backbone.

Secondly, we used the heterogeneous counterpart PCN-224(Rh) as catalyst under the same reaction conditions as above and found a similar yield ( $29 \%$ after $22 \mathrm{~h}$ ). The selectivity towards the cyclopropanation products was decreased due to the uncontrolled dimerization of EDA (found by GC-MS and ${ }^{1} \mathrm{H}$ and ${ }^{13} \mathrm{C}$ NMR analysis); EDA is therfore no longer available for the carbene transfer to the olefin. Therefore, the reaction was performed using a motorized pump and excess of olefin conditions; hereby, $135 \mu \mathrm{L}(1.1 \mathrm{mmol})$ EDA diluted in $3 \mathrm{~mL}$ $\mathrm{CH}_{2} \mathrm{Cl}_{2}$ was added at a rate of $0.5 \mathrm{~mL} / \mathrm{h}$ to a suspension of catalyst in $2 \mathrm{~mL}$ olefin solution. As a result, a conversion of $71 \%$ styrene and a $d r$ of 1:1 (trans:cis) was observed; formation of the coupling side product diethyl fumarate was drastically reduced, revealing the crucial dependence of controlled introduction of EDA on the yield of the reaction.

Furthermore, we determined the scope of the reaction, as summarized in Table 1. Products of the substituted 4-methyl-, 4-methoxy-, 4-nitro-, 4-chlorostyrenes were obtained in moderate to good yields up to $68 \%$ at r.t. after $6 \mathrm{~h}$ under stirring under air applying excess substrate. Cyclic and terminal olefins exhibited yields of $49 \%$ (cyclooctene) and $22 \%$ (1-octene). In addition, 3,4-dihydro-2H-pyran, a sugar-based glycal, which can be obtained from biological feedstock which makes it relevant in terms of sustainability, showed a moderate yield of $20 \%$. 
Table 1. Catalytic results for the cyclopropanation of olefins and ethyl diazoacetate (EDA) using MP-MOFs as catalyst.

\begin{tabular}{lllll}
\hline No. & Olefin & Catalyst $^{1}$ & $\begin{array}{l}\text { Yield }^{3} \\
{[\%]}\end{array}$ & $d r^{4}$ \\
\hline 1 & Styrene & PCN-224(Rh) & 71 & $1: 1$ \\
2 & Styrene & \\
3 & Styrene & PCN-224(Rh) & 29 & $1: 1$ \\
4 & 4-Methylstyrene & Rh-TCCPOMe & 20 & $1: 1$ \\
5 & 4-Methoxystyrene & PCN-224(Rh) & 58 & $1: 1$ \\
6 & 4-Nitrostyrene & PCN-224(Rh) & 52 & $1: 1$ \\
7 & 4-Chlorostyrene & PCN-224(Rh) & 68 & $1: 1$ \\
8 & 4-Aminostyrene & PCN-224(Rh) & 35 & $1: 1$ \\
9 & 4-Aminostyrene (AS) & PCN-222(Rh) & 29 & $42: 1$ \\
10 & 4-Hydroxystyrene & PCN-224(Rh) & 4 & $21: 1$ \\
11 & N,N-Dimethyl-4-AS & PCN-224(Rh) & 31 & $1.5: 1$ \\
12 & N,N-Dimethyl-4-AS & PCN-222(Rh) & 16 & $1: 1$ \\
13 & 1-Octene & PCN-224(Rh) & 22 & $1: 1$ \\
14 & Cyclooctene & PCN-224(Rh) & 49 & $1: 1$ \\
15 & 3,4-Dihydro-2H-pyran & PCN-224(Rh) & 20 & $1: 1$ \\
\hline
\end{tabular}

All reactions were carried out at room temperature and stirring $(\mathrm{rpm}=500)$ operated under air after $6 \mathrm{~h}$ reaction time. Yields are obtained applying an excess of substrate: $135 \mu \mathrm{L}(1.1 \mathrm{mmol}) \mathrm{EDA}$ diluted in $3 \mathrm{~mL} \mathrm{CH} \mathrm{Cl}_{2}$ was added via a motorized syringe pump (rate $=0.5 \mathrm{~mL} / \mathrm{h}$ ) to a suspension of catalyst in $2 \mathrm{~mL}$ substrate. ${ }^{1}$ Catalyst loading: $0.0033 \mathrm{mmol}, 0.4 \mathrm{~mol} \% \mathrm{Rh}$. ${ }^{2}$ Manual addition of $135 \mu \mathrm{LEDA}$ (1.1 mmol) in $2 \mathrm{~mL} \mathrm{CH} \mathrm{Cl}_{2}$ to a solution of $75 \mu \mathrm{L}$ styrene in $2 \mathrm{~mL} \mathrm{CH}_{2} \mathrm{Cl}_{2}$ within 20 min. ${ }^{3} \mathrm{GC}$-Yield. ${ }^{4}$ Diastereomeric ratio (trans:cis) of the product.

Interestingly, a high $d r$ of 23:1 (trans:cis) was observed with 4aminostyrene as substrate. Presumably, the amino functionality acts as an anchoring ligand to the Rh-porphyrin linkers and/or via hydrogen bonding to the $\mathrm{Zr}$-oxo-clusters, as it is indicated by infrared spectroscopy (see the ESI, Figures S8 and S9). Such a coordination mode is likely to align the substrate in a certain position, and sterically orienting the olefin moiety to the Rhcarbene in a favored fashion. In this sense, the reaction can be considered catalyst-controlled, because of the well-defined and rigid $\mathrm{Rh}(\mathrm{Zr})$ - $\mathrm{Rh}$ distances favoring certain olefin orientations. On the other hand, the reaction can be viewed as substratecontrolled, whereby the coordination ability of functional groups on the substrates drives the stereoselectivity. In this example, the linkers and/or nodes carrying functional groups may act as binding sites facilitating substrate-catalyst interactions for possible enrichment fixation, and activation of substrates, providing special transfer pathways for stereoselective chemical transformation. ${ }^{48}$ Hence, coordination of the substrate caused by the pore confinement results in its symmetry breaking, with the carbene transfer proceeding no longer statistically but favorably towards one diastereomer over the other. The high diastereoselectivity thus follows from stabilization of an alternative transition state, compared to molecular Rh-porphyrins in solution, which naturally lack any pore confinement effect or substrate-controlled preferential steric orientation in the transition state of the carbene transfer. The abovementioned stabilization is also known from cagecatalyzed reactions leading to unusual reactivity/selectivity compared to their bulk counterparts. ${ }^{10,49,50}$
Further, we investigated the conversion of 4-hydroxystyrene and we found a similarly high $d r$ of 21:1 (trans:cis), which supports the idea of the directing group effect of the substrate. However, the yield of the corresponding cyclopropane was low due to side reactions with propylene glycol, which is present in the solution of 4-hydroxystyrene (10 wt\%), acting as a stabilizer. As a control experiment to test that the diastereoselectivity is controlled by coordinating groups attached to the styrene, we further investigated $\mathrm{N}, \mathrm{N}$-dimethyl4-aminostyrene and 4-methoxystyrene. In both substrates, the coordination ability is reduced by sterical hindrance the absence of $\mathrm{H}$-bonding interactions. In neither case did we observe diastereoselective transformations, while the product yield for $\mathrm{N}, \mathrm{N}$-dimethyl-4-aminostyrene was consistent with that of 4-aminostyrene (4-methoxystyrene does not contain a stabilizer, hence a direct comparison of yields cannot be made). In contrast to other substrate-directed reactions which often proceed via an intramolecular pathway, the ascribed reaction is intermolecular, since coordination and catalytic reactions do proceed at two distinct centers. When we consider the coordination of the amino group to a Rh-porphyrin, the Rhporphyrin may have two important different functions: (1) Coordination acceptor and pre-alignment of non-innocent substrate and (2) catalytically active center. This interplay of two neighboring Rh-centers may critically influence the diastereoselectivity of the reaction.

\subsection{Topology-dependent diastereoselectivity}

To investigate the interplay of two neighboring Rh-porphyrins as a function of the Rh-Rh distances, we studied PCN-222(Rh), which consists out of the same building units as PCN-224(Rh) but differs in pore geometry and topology exhibiting small trigonal and large hexagonal pores. In contrast to a Rh-Rh distance of $13.6 \AA$ found in PCN-224(Rh), PCN-222(Rh) provides smaller Rh-Rh distances of $9.7 \AA$ within its smaller trigonal micropores.
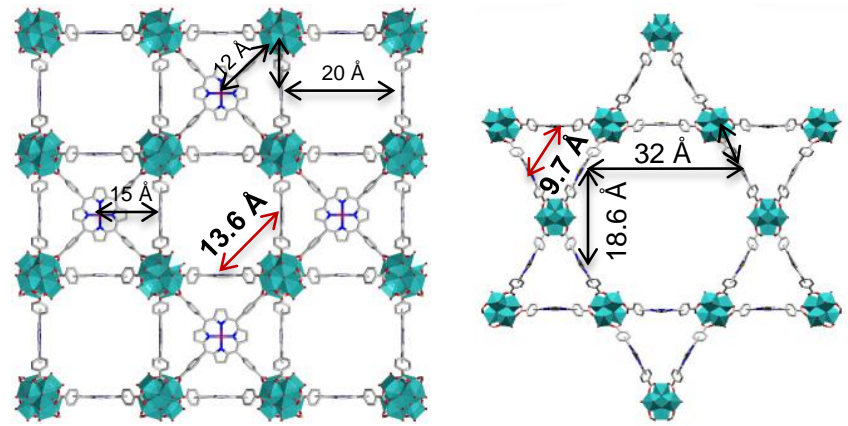

Figure 4. Schematic representation of PCN-224(Rh) (left) exhibiting squared micropores and PCN-222(Rh) revealing its small trigonal micropores and large hexagonal mesopores (right). Black and bold arrows indicate Rh-Rh distances, while black arrows represent $\mathrm{Zr}-\mathrm{Rh}$ distances, respectively. Red arrows highligh the most feasible (shortest possible) Rh-Rh distances, which would favor the

4-Aminostyrene has a dimension of $8.1 \AA$ and when we consider a bond length of around $2 \AA$ for Rh-carbene, ${ }^{51}$ a cumulative length of $\sim 10 \AA$ is obtained. Hence, the summed distance of substrate and carbene matches quite well with the $R h-R h$ distance of $9.7 \AA$ in $\mathrm{PCN}-222(\mathrm{Rh})$. Indeed, reaction of EDA and 
4-aminostyrene proceeds with a moderate yield of $29 \%$, but with an exceptional $d r$ of 42:1 (trans:cis). The small trigonal pore is built from three distinct $\mathrm{Rh}$-centers with identical $\mathrm{Rh}-\mathrm{Rh}$ distances. Thus, in our view, this specific micro environment confines the reaction space and makes it more likely that these two species will react in very selective manner, leading to strongly enhanced diastereoselectivity (see the SI, Figure S7 for qualitative graphic illustrations highlighting the possible orientations of 4-aminostyrene inside a cavity of PCN-222(Rh)). Since the $\mathrm{Zr}-\mathrm{Rh}$ distances in both MOFs are identical and the major differences in the Kagomé structure of PCN-222(Rh) are the shorter $\mathrm{Rh}-\mathrm{Rh}$ distances, we believe that the role of $\mathrm{H}$ bonding interactions of amino or hydroxy groups with the $\mathrm{Zr}$ oxo clusters are not that dominant. However, we cannot exclude nor quantify the contribution of $\mathrm{H}$-bonding interactions. In a conceptually similar study Zhang et al. applied Zn-BCTA MOF (BCTA = bis[4-(5-carboxy-2-thienyl)phenyl] (4carboxyphenyl)amine) as a photocatalyst in the sulfonylationcyclization of activated alkenes. ${ }^{52}$ Interpenetration of the MOF allowed closer substrate-redox center contacts, resulting in superior efficiency and more importantly, in higher diastereoselectivities compared to the homogeneous counterpart. ${ }^{52}$ These results are in line with the obtained diastereoselectivities in our work, which also critically depend on confinement effects and suitable substrate-catalyst distances.

\section{Conclusions}

We introduced an example of a catalytic process wherein the diastereoselectivity crucially depends on specific local confinement effects, which can be adjusted by the careful choice of an appropriate MOF system. For the first time, heterogeneous porphyrin-based metal-organic frameworks PCN-222(Rh) and PCN-224(Rh) catalysts were applied in the diastereoselective cyclopropanation of styrene and styrene derivatives with ethyl diazoacetate. Styrene and substituted styrenes were converted with high catalytic activity. Interestingly, styrenes carrying coordinating amino and hydroxy groups show a high diastereomeric ratio $(d r)$ of up to 23:1 (trans:cis) under PCN-224(Rh) catalyst, which was attributed to their coordination to Rh centers caused by pore confinement effects derived from favorable substrate-catalyst interactions. The diastereoselectivity is further improved to a $d r$ of 42:1 (trans:cis) by selecting PCN-222(Rh) catalyst, a structural analog of PCN-224 with Kagomé topology, featuring closer Rh-Rh distances. These results demonstrate that the diastereoselectivity of the cyclopropanation reaction can be controlled by (1) the choice of functional groups attached to substrates and (2) by structural and topological differences of the two MOFs used in this study, i.e. the distances between two rhodium centers. The insights obtained in this work may be interesting for the development of novel MOFs catalysts, since the results showcase possible structure-property relationships and more importantly, stress the impact of confined space as a critical reaction parameter to obtain stereoselective reaction products.

\section{Conflicts of interest}

There are no conflicts to declare.

\section{Acknowledgements}

The authors thank Werner Heinz for his assistance and fruitful discussions. BB is grateful to the Research Foundation Flanders (FWO) for funding (post-doctoral grant). DDV thanks FWO for project funding, and KU Leuven (Metusalem grant). This work was funded by the European Union's Horizon 2020 research and innovation program under the Marie SklodowskaCurie grant agreement no. 641887 (project acronym: DEFNET).

\section{Notes and references}

1. R. Noyori, Angew. Chem. Int. Ed., 2002, 41, 2008-2022.

2. S. F. Kirsch, Angew. Chem. Int. Ed., 2009, 48, 2450-2451.

3. A. Miyashita, A. Yasuda, H. Takaya, K. Toriumi, T. Ito, T. Souchi and R. Noyori, J. Am. Chem. Soc., 1980, 102, 7932-7934.

4. T. Katsuki and K. B. Sharpless, J. Am. Chem. Soc., 1980, 102, 5974-5976.

5. K. Manna, T. Zhang and W. Lin, J. Am. Chem. Soc., 2014, 136, 6566-6569.

6. J. S. Seo, D. Whang, H. Lee, S. I. Jun, J. Oh, Y. J. Jeon and K. Kim, Nature, 2000, 404, 982-986.

7. A. Gheorghe, M. A. Tepaske and S. Tanase, Inorg. Chem. Front., 2018, 5, 1512-1523.

8. M. Yoon, R. Srirambalaji and K. Kim, Chem. Rev., 2012, 112, 1196-1231.

9. S. H. A. M. Leenders, R. Gramage-Doria, B. de Bruin and J. N. H. Reek, Chem. Soc. Rev., 2015, 44, 433-448.

10. A. M. Castilla, W. J. Ramsay and J. R. Nitschke, Acc. Chem. Res., 2014, 47, 2063-2073.

11. J. C. Jansen, E. J. Creyghton, S. L. Njo, H. van Koningsveld and H. van Bekkum, Catal. Today, 1997, 38, 205-212.

12. A. Corma, M. E. Domine, L. Nemeth and S. Valencia, J. Am Chem. Soc., 2002, 124, 3194-3195.

13. C. Li, H. Zhang, D. Jiang and Q. Yang, Chem. Comm., 2007, 547558.

14. S. H. Kim, C. K. Shin, J. H. Seok, C. Y. Lee and G. J. Kim, in Stud. Surf. Sci. Catal., 2007, 165, 745-748.

15. A. Corma, M. Iglesias, F. X. L. i. Xamena and F. Sánchez, Chem Eur. J., 2010, 16, 9789-9795.

16. F. G. Cirujano, F. X. Llabrés i Xamena and A. Corma, Dalton Trans., 2012, 41, 4249-4254.

17. V. L. Rechac, F. G. Cirujano, A. Corma and F. X. Llabrés i Xamena, Eur. J. Inorg. Chem., 2016, 4512-4516.

18. H. H. Mautschke, F. Drache, I. Senkovska, S. Kaskel and F. X. Llabrés i Xamena, Catal. Sci. Technol., 2018, 8, 3610-3616.

19. R. E. Morris and X. Bu, Nat. Chem., 2010, 2, 353-361.

20. T. Sawano and H. Yamamoto, J. Org. Chem., 2018, 83, 48894904.

21. K. Epp, A. L. Semrau, M. Cokoja and R. A. Fischer, ChemCatChem, 2018, 10, 3506-3512.

22. C. G. Hamaker, G. A. Mirafzal and L. K. Woo, Organometallics, 2001, 20, 5171-5176.

23. P. Tagliatesta and A. Pastorini, J. Mol. Catal. A: Chem., 2003, 198, 57-61. 
24. Y. Chen, J. V. Ruppel and X. P. Zhang, J. Am. Chem. Soc., 2007, 129, 12074-12075.

25. H. J. Callot and C. Piechocki, Tetrahedron Lett., 1980, 21, 34893492.

26. S. O'Malley and T. Kodadek, Organometallics, 1992, 11, 22992302.

27. A. G. M. Barrett, D. C. Braddock, I. Lenoir and H. Tone, J. Org. Chem., 2001, 66, 8260-8263.

28. M. Frauenkron and A. Berkessel, Tetrahedron Lett., 1997, 38, 7175-7176.

29. J.-C. Wang, Y. Zhang, Z.-J. Xu, V. K.-Y. Lo and C.-M. Che, ACS Catal., 2013, 3, 1144-1148.

30. D. A. Smith, D. N. Reynolds and L. K. Woo, J. Am. Chem. Soc., 1993, 115, 2511-2513.

31. Z. Gross, N. Galili and L. Simkhovich, Tetrahedron Lett., 1999, 40, 1571-1574.

32. D. Intrieri, A. Caselli and E. Gallo, Eur. J. Inorg. Chem., 2011, 5071-5081.

33. D. M. Carminati, D. Intrieri, A. Caselli, S. L. Gac, B. Boitrel, L. Toma, L. Legnani and E. Gallo, Chem. Eur. J., 2016, 22, 1359913612.

34. L. Huang, Y. Chen, G.-Y. Gao and X. P. Zhang, J. Org. Chem., 2003, 68, 8179-8184.

35. S. Fantauzzi, E. Gallo, E. Rose, N. Raoul, A. Caselli, S. Issa, F. Ragaini and S. Cenini, Organometallics, 2008, 27, 6143-6151.

36. S. Zhu, J. V. Ruppel, H. Lu, L. Wojtas and X. P. Zhang, J. Am. Chem. Soc., 2008, 130, 5042-5043.

37. D. Feng, Z.-Y. Gu, J.-R. Li, H.-L. Jiang, Z. Wei and H.-C. Zhou, Angew. Chem. Int. Ed., 2012, 51, 10307-10310.

38. D. Feng, W.-C. Chung, Z. Wei, Z.-Y. Gu, H.-L. Jiang, Y.-P. Chen, D. J. Darensbourg and H.-C. Zhou, J. Am. Chem. Soc., 2013, 135, 17105-17110.

39. W. Morris, B. Volosskiy, S. Demir, F. Gándara, P. L. McGrier, H. Furukawa, D. Cascio, J. F. Stoddart and O. M. Yaghi, Inorg. Chem., 2012, 51, 6443-6445.

40. H. Lebel, J.-F. Marcoux, C. Molinaro and A. B. Charette, Chem. Rev., 2003, 103, 977-1050.

41. C. Ebner and E. M. Carreira, Chem. Rev., 2017, 117, 1165111679.

42. G. Maas, Chem. Soc. Rev., 2004, 33, 183-190.

43. Carbocyclic Three-Membered Ring Compounds: Cyclopropanes, Transformations; Houblen-Weyl Methods of Organic Chemistry, Stuttgart, Germany, 1997.

44. H. J. Callot, F. Metz and C. Piechocki, Tetrahedron, 1982, 38, 2365-2369.

45. M. P. Doyle, W. R. Winchester, J. A. A. Hoorn, V. Lynch, S. H. Simonsen and R. Ghosh, J. Am. Chem. Soc., 1993, 115, 99689978.

46. J. Liu, Y.-Z. Fan, X. Li, Z. Wei, Y.-W. Xu, L. Zhang and C.-Y. Su, Appl. Catal., B, 2018, 231, 173-181.

47. F. Rouquerol, J. Rouquerol and K. S. W. Sing, Adsorption by Powders and Porous Solids: Principles, Methodology and Applications, San Diego, USA, 1999.

48. X. Jing, C. He, L. Zhao and C. Duan, Acc. Chem. Res., 2019, 52, 100-109.

49. J. D. Bass, S. L. Anderson and A. Katz, Angew. Chem. Int. Ed., 2003, 42, 5219-5222.

50. J. L. Bolliger, in Effects of Nanoconfinement on Catalysis, ed. R. Poli, Springer International Publishing, Cham, 2017, DOI: 10.1007/978-3-319-50207-6_2, pp. 17-48.

51. L. R. Collins, M. van Gastel, F. Neese and A. Fürstner, J. Am. Chem. Soc., 2018, 140, 13042-13055.
52. T. Zhang, Y. Shi, S. Zhang, C. Jia, C. He and C. Duan, New J. Chem., 2018, 42, 18448-18457. 